\title{
IL-1 receptor type 1-knock-out mice fed a high-fat diet for 16 weeks become obese but remain insulin sensitive
}

\author{
E. Oliver ${ }^{1}$, M. Claessens ${ }^{1}$, K. Harford ${ }^{1}$, C. Reynolds ${ }^{1}$, K. H. G. Mills ${ }^{2}$ and H. M. Roche ${ }^{1}$ \\ ${ }^{1}$ Nutrigenomics Research Group, UCD Conway Institute, University College Dublin, Republic of Ireland and \\ ${ }^{2}$ Immune Regulation Research Group, Trinity College Dublin, Republic of Ireland
}

Obesity is the key aetiology factor that predisposes individuals to insulin resistance. Obesity leads to a sub-acute pro-inflammatory state with infiltration of macrophages into adipose tissue ${ }^{(1)}$. It is proposed that adipose tissue macrophages release pro-inflammatory mediators impeding adipocyte insulin signalling, ultimately resulting in insulin resistance. Previous work has demonstrated that IL-1 receptor type I-knock-out (IL-1 $\mathrm{R}^{-l^{-}}$) mice are protected against obesity-induced insulin resistance following a high-fat diet (HFD). The present timecourse study aimed to investigate when obesity-induced insulin resistance develops in C57Bl/6 wild type (WT) mice and compare this HFD-induced phenotype in IL-1RI ${ }^{-1-}$ mice.

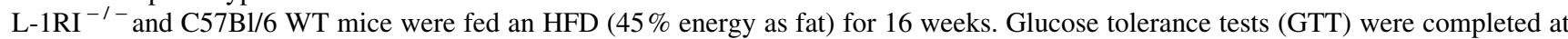
0 ( $n$ 8), 4 (n 6), 6 ( $n$ 6, C57B1/6 WT group; $n$ 5, IL-1RI ${ }^{-\prime-}$ group), 12 ( $n$ 6) and 16 ( $n$ 8, C57B1/6 WT group; $n$ 4, IL-1RI ${ }^{-1-}$ group) $^{-1}$ weeks. Fasting plasma glucose, insulin, TAG and NEFA concentrations were determined at 0,6 and 12 weeks. Throughout the study body weight and food intake were recorded. Repeated measures ANOVA identified significant differences in the GTT between C57B1/6 WT and IL-1RI ${ }^{-1-}$ mice over time. One-way ANOVA determined significant differences in fasting metabolic markers within and between groups during the time-course study.

At weeks $6,8,10$, and 12 the C57BL/6 WT mice gained significantly more weight than the IL-1R $1^{-/-}$mice; however, by week 14 there was no significant difference between body weights. Interestingly, at weeks 12 and 16 the IL-1R $1^{-/-}$mice had significantly lower epididymal adipose tissue and visceral adipose tissue mass. Furthermore, the IL-1R1 ${ }^{-1}$ mice had higher energy intake at weeks 8,12 and 16 compared with the C57BL/6 WT mice. In the C57BL/6 WT mice there was a clear time-dependent deterioration in insulin sensitivity from week 6 onwards compared with week 0 . Initially, at week 0 the IL-1R1 ${ }^{-1}$ mice had a higher glucose peak, although clearing the glucose effectively by $120 \mathrm{~min}$ as compared with the C57BL/6 WT mice. At week 4 and 6 the IL-1R1 ${ }^{-1-}$ mice cleared glucose more effectively than the C57BL/6 WT mice; however, at week 12 the IL-1R1 ${ }^{-1}$ mice seemed to have reached a point of insulin resistance similar to the C57BL/6 WT mice. Interestingly, at week 16 the IL-1R $1^{-/}$mice seemed to become more insulin sensitive compared with the C57BL/6 WT mice since they cleared glucose more effectively. The IL-1R1 ${ }^{-/}$mice had significantly lower fasting insulin levels by week 6 compared with C57BL/6 WT mice. Fasting NEFA concentrations were significantly higher in the C57BL/6 WT compared to the IL-1R1 $1^{-/-}$mice at week 16 and TAG levels were significantly higher at weeks 6 and 16 in the C57BL/6 WT mice compared with the IL-1R1 ${ }^{-/-}$mice. Fasting plasma leptin levels increased over time in the C57BL/6 WT mice while the IL-1R1 ${ }^{-/-}$mice showed lower levels of leptin and adiponectin throughout the study.

The present study shows that IL-1RI ${ }^{-1}$ mice are partly protected from obesity-induced insulin resistance by progressing more slowly to an insulin-resistant state. Further work is continuing to establish the specific molecular mechanisms involved in this protection.

This work was funded by Science Foundation Ireland, Principal Investigator Programme. E. O. was supported by the HRB PhD Training Site Programme at Trinity College Dublin.

1. Weisberg S, McCann D, Desai M et al. (2003) J Clin Invest 112, 1796-1808. 